\title{
Diacronie
}

Studi di Storia Contemporanea

$\mathrm{N}^{\circ} 30,2$ | 2017

Ponti fra nazioni e continenti

\section{Edoardo Grassia, Sabato Martelli Castaldi. Il generale partigiano}

\section{Matteo Tomasoni}

\section{Q OpenEdition \\ 1 Journals}

Edizione digitale

URL: http://journals.openedition.org/diacronie/5941

DOI: $10.4000 /$ diacronie.5941

ISSN: 2038-0925

Editore

Association culturelle Diacronie

Notizia bibliografica digitale

Matteo Tomasoni, «Edoardo Grassia, Sabato Martelli Castaldi. II generale partigiano », Diacronie [Online], $N^{\circ} 30,2$ | 2017, documento 10, Messo online il 29 juillet 2017, consultato il 23 septembre 2020. URL : http://journals.openedition.org/diacronie/5941 ; DOI : https://doi.org/10.4000/diacronie.5941 


\title{
Diacronie
}

Studi di Storia Contemporanea

30, 2/2017

Ponti fra nazioni e continenti: diplomazia, immaginari e conoscenze tecniche

\section{RECENSIONE: Edoardo GRASSIA, Sabato Martelli Castaldi. Il generale partigiano, Milano, Mursia, 2016, 338 pp.}

\author{
A cura di Matteo TOMASONI
}

Per citare questo articolo:

TOMASONI, Matteo, «RECENSIONE: Edoardo GRASSIA, Sabato Martelli Castaldi. Il generale partigiano, Milano, Mursia, 2016, 338 pp.», Diacronie. Studi di Storia Contemporanea : Ponti fra nazioni e continenti: diplomazia, immaginari e conoscenze tecniche, 30, 2/2017, 29/7/2017,

URL: < http://www.studistorici.com/2017/07/29/tomasoni_numero_30/ >

Diacronie Studi di Storia Contemporanea $\rightarrow$ http://www.diacronie.it

Rivista storica online. Uscita trimestrale.

redazione.diacronie@hotmail.it

Comitato di direzione: Naor Ben-Yehoyada - João Fábio Bertonha - Christopher Denis-Delacour - Maximiliano Fuentes Codera Anders Granås Kjøstvedt - John Paul Newman - Deborah Paci - Niccolò Pianciola - Spyridon Ploumidis - Wilko Graf Von Hardenberg

Comitato di redazione: Jacopo Bassi - Luca Bufarale - Gianluca Canè - Fausto Pietrancosta - Alessandro Salvador - Matteo Tomasoni Diritti: gli articoli di Diacronie. Studi di Storia Contemporanea sono pubblicati sotto licenza Creative Commons 3.0. Possono essere riprodotti e modificati a patto di indicare eventuali modifiche dei contenuti, di riconoscere la paternità dell'opera e di condividerla allo stesso modo. La citazione di estratti è comunque sempre autorizzata, nei limiti previsti dalla legge. 


\title{
10/ RECENSIONE: Edoardo GRASSIA, Sabato Martelli Castaldi. Il generale partigiano, Milano, Mursia, 2016, 338 pp.
}

\author{
A cura di Edoardo GRASSIA
}

Quando l'8 settembre del 1985 in una solenne cerimonia in Campidoglio venne conferita postuma al generale Sabato Martelli Castaldi la Medaglia d'oro al valore militare, pochi sapevano i retroscena e le vicissitudini di questo personaggio. Fu solo nell'anno 1996 quando lo storico e giornalista Mario Avagliano pubblicò un testo ${ }^{1}$ che, a distanza di più di cinquant'anni dalla sua morte, prese in considerazione quell'«esempio nobilissimo di completa e disinteressata dedizione alla causa della libertà del proprio Paese $»^{2}$ - così come si dichiarava nell'onorificenza conferita pochi anni prima - che venne riscoperta questa figura. Ma la vita di Sabato Martelli fu ben più complessa ed articolata della sola adesione alla Resistenza romana, così come dimostrato dalla sua partecipazione nella Prima guerra mondiale in veste di ufficiale, dalla sua rapida carriera nell' Aeronautica, dall'iniziale avvicinamento al fascismo ma anche dal suo rapido disinganno e dal graduale allontanamento da Mussolini.

Il merito di aver ridato "voce" a questo poco conosciuto protagonista della storia militare italiana del XX secolo spetta allo storico Edoardo Grassia, il quale ha saputo ricostruire con precisione e caparbietà un difficile capitolo del periodo interbellico. Parlare di Sabato Martelli non vuol dire limitarsi ai suoi anni come ufficiale dell'Aeronautica, né al suo particolare rapporto con l'«esuberante, irrequieto e amante della vita»3 Italo Balbo - quest'ultimo nominato Ministro dell'Aeronautica proprio da Mussolini nel 1929 - ma nemmeno ridurre la sua esperienza politica agli anni della Resistenza, quando si fece conoscere con il nome di battaglia "Tevere". Nell'introduzione al testo l'autore ci informa della particolarità del personaggio, ma soprattutto avvisa della necessità di retrocedere nel passato proprio perché il generale Martelli deve inserirsi tra i limiti imposti dalla Grande Guerra sino alla Resistenza, passando attraverso uno dei momenti

\footnotetext{
${ }^{1}$ AVAGLIANO, Mario, Il partigiano Tevere. Il generale Sabato Martelli Castaldi dalle vie dell'aria alle Fosse Ardeatine, Cava de' Tirreni, Avagliano Editore, 1996.

${ }^{2}$ PRESIDENZA DELLA REPUBBLICA, Martelli Castaldi, Sabato - Medaglia d'oro al valor militare, decreto del 1944 (cerimonia di consegna postuma, 1985); il testo completo della motivazione in URL: < http://www.quirinale.it/elementi/DettaglioOnorificenze.aspx?decorato=13318 > [consultato il 15 febbraio 2017].

${ }^{3}$ Cfr. CIANO, Galeazzo, Diario 1937-1943, Milano, Rizzoli, 1980, p. 447, nota del 29 giugno 1940.
} 
decisivi della storia italiana di quell'epoca: il fascismo. Fondamentali sono stati i colloqui che l'autore ha mantenuto con gli eredi della famiglia, ma anche le frequenti visite ai numerosi archivi elencati nell'opera, fra cui si distinguono quelli militari e particolarmente l'Ufficio Storico dell'Aeronautica dove da tempo Grassia collabora nell'ambito della ricerca storica.

L'autore dà inizio alla sua opera partendo da un episodio fondamentale della vita di Sabato Martelli e cioè il primo conflitto mondiale. Durante questo periodo il giovane ufficiale sperimentò e condivise in prima persona la drammatica esperienza della guerra insieme a colui che sarà suo amico "sino alla fine", Roberto Lordi, ma conobbe anche l'affascinante modernità dell'esperienza bellica che lo spinse - con sempre più interesse - ad avvicinarsi alla neonata Aeronautica militare. Ben presto, già nell'aprile del 1917, Martelli abbandonò le trincee dell'Isonzo per spostarsi nei cieli del Carso per poi - dopo la disastrosa sconfitta di Caporetto - partecipare alla guerra aerea sopra il Tagliamento ed il Piave. Finì il conflitto nei cieli della Libia, dove continuò sino al 1919 combattendo contro la resistenza delle tribù locali.

Il seguente e "logico" passo nella vita di Sabato Martelli fu la sua adesione al Partito Nazionale Fascista, attraverso un gruppo di ex-combattenti che finalmente lo portò ad essere una figura di rilievo all'interno della Regia Aeronautica. La sua carriera fu fulminea, così come il suo allontanamento dal corpo: se nel 1931 ricoprì l'incarico di Capo Gabinetto del ministro Italo Balbo e nel 1933 venne addirittura nominato come il più giovane generale d'Italia, il suo stretto legame con i "balbisti" sarebbe poi stato considerato inopportuno dal Duce che non esitò a decretare - nel novembre del 1935 - un "riposo forzato" del giovane generale.

Lodevole, in questo senso, è il lavoro dello storico Edoardo Grassia il quale, attraverso la ricostruzione delle esperienze politiche e militari del generale Martelli, riesce anche a proporre un'analisi più sostanziale e quasi intima del personaggio nelle fasi più delicate della sia vita. La risolutezza dell'ex-generale nel voler scoprire la verità sulla complicata (e spesso contrastante) relazione tra Balbo e Mussolini fu, con ogni probabilità, la causa di molti dei problemi che Martelli dovette affrontare durante i successivi dieci anni: dal congedo forzato, al lavoro precario, alle minacce, al controllo da parte dell'OVRA (la polizia segreta fascista) fino alla vera e propria persecuzione dopo l'8 settembre 1943. L'autore è stato particolarmente attento a quest'ultima esperienza; non solo perché si tratta di un periodo nel quale l'ex-generale visse sotto l'attento controllo delle autorità nazifasciste, ma anche per la sua minuziosa descrizione degli eventi l'occupazione di Roma, l'organizzazione partigiana, i rastrellamenti, gli attentati, la vita in carcere, ecc. - che caratterizzarono quel preciso periodo storico: dal settembre del 1943 al 23 marzo 1944, giorno della sua morte.

Sebbene Grassia riferisce che sin dall'ottobre del 1943 Martelli Castaldi aderì con lucidità alla Resistenza, è anche vero che i primi mesi dell'occupazione furono dedicati ad un pericolosissimo “doppio gioco" che l'ex-generale portò avanti finché gli fu possibile. La sua esperienza come 
militare, i suoi numerosi contatti anche nei servizi di sicurezza o le storiche amicizie nell'esercito furono fondamentali per il suo lavoro di informatore per i membri della Resistenza. Martelli fornì indicazioni riservate che furono essenziali per l'organizzazione e l'operato della lotta antifascista romana, specialmente durante le prime concitate fasi dell'occupazione tedesca portata a termine dal feldmaresciallo Albert Kesselring. Eppure Sabato Martelli fu anche un uomo d'azione così come lo era stato durante il primo conflitto: non esitò a prender parte insieme al Fronte Militare Clandestino (FMC) e ad altre formazioni partigiane - in cui militavano personaggi politici di spicco come Emilio Lussu, Giordano Amidani o Mario Colacchi (tutti esponenti del CLN fondato a Roma proprio in quei giorni) - ai primi combattimenti di Porta San Paolo, della Montagnola ma anche di Porta San Giovanni sino alla dolorosa resa del 10 settembre. Secondo la ricostruzione di Grassia, la collaborazione con il FMC fu indubbiamente frenetica: Martelli rischiò numerose volte la vita per estrarre armi da alcuni edifici presidiati della capitale occupata, fra cui - lo conosceva particolarmente bene - la sede del Ministero dell'Aeronautica; oppure nei vari tentativi di assistere altri partigiani impegnati in missioni che avevano l'obiettivo di ostacolare le autorità nazifasciste in città.

Nonostante le precauzioni e la certezza di non mettere a repentaglio la vita dei suoi familiari, Sabato Martelli fu uno dei tanti partigiani che caddero nelle mani dei tedeschi durante un rastrellamento realizzato dalla Schutzstaffeln di Herbert Kappler, responsabile dell'ordine cittadino. È proprio qui che l'autore ricostruisce al dettaglio - con i pochi documenti disponibili non solo l'arresto e l'immediata reclusione di Sabato Martelli nell'improvvisato carcere di via Tasso (17 gennaio 1944), ma anche la drammatica esperienza di quei pochi ma durissimi mesi di prigionia. In quell'oscuro luogo, un vecchio palazzo signorile nel pieno centro di Roma, Sabato e l'inseparabile amico Roberto Lordi passarono gli ultimi giorni della loro vita nella speranza che gli Alleati portassero a termine l'operazione militare che da Anzio (22 gennaio) doveva, nel giro di pochi giorni, portare alla liberazione di Roma (Operation Shingle). Come ben sappiamo ci vollero però quasi cinque mesi per vedere i mezzi corazzati statunitensi transitare per le vie della capitale, motivo per il quale l'unica resistenza al controllo nazifascista in città fu ancora una volta portata avanti dai partigiani i quali combattevano con le poche risorse a loro disposizione.

Il 23 marzo del 1944 un attentato in via Rasella provocò la morte di trentasette militari, molti di essi appartenenti al SS-Polizeiregiment "Bozen" composta da sudtirolesi inquadrati nella polizia tedesca. Questo fatto segnò, il giorno dopo, la condanna a morte di oltre trecento persone fra cui vi erano ebrei della comunità romana, alcuni civili legati all'attentato, partigiani e membri della Resistenza prelevati dal carcere di via Tasso e da quello di Regina Coeli. La rappresaglia fu voluta dallo stesso Hitler come avvertimento per la popolazione civile, ma ancora oggi stupiscono la freddezza e la celerità nella pianificazione di un crimine di queste proporzioni, portato a termine nel giro di poche ore lo stesso 24 marzo. Fra i criminali di guerra che furono condannati dai 
tribunali militari per l'Eccidio delle Fosse Ardeatine, ricordiamo i già citati Albert Kesselring e Herbert Kappler, ma anche Kurt Mälzer, Eberhard von Mackensen e l'ufficiale Erich Priebke (aiutante di Kappler), molti dei quali però finirono per scontare pene minime nonostante la loro diretta partecipazione a questi crimini di guerra.

Sabato Martelli, così come l'amico Roberto Lordi e molti altri dirigenti della Resistenza romana, venne assassinato con un colpo alla nuca nelle fosse allora conosciute come "cave Ardeatine" proprio quel 24 marzo del 1944. Pochi mesi dopo, con la città già liberata, si procedette al recupero delle salme fra le quali si poté ancora riconoscere la maggior parte delle persone lì selvaggiamente uccise. L'autore, meticoloso sino alla fine del suo lavoro, indica che il cadavere dell'ex-generale e partigiano “Tevere" fu riconosciuto e deposto nel sarcofago $\mathrm{n}^{\mathrm{o}}$ 115, per poi essere seppellito nel mausoleo costruito all'interno delle Fosse inaugurato dal Capo dello Stato, Enrico de Nicola, nel marzo del 1948.

La storia di Sabato Martelli Castaldi è una delle tante pagine dimenticate della storia italiana; una figura a lungo omessa dalla storiografia nazionale in quanto vittima - suo malgrado - di quello che l'autore chiama esser stata una parabola tragica ed allo stesso tempo eroica della sua vita $^{4}$. Eppure, l'obiettivo di quest'opera è proprio quella di riconsegnare ad ognuno di noi la memoria di una persona qualunque che, l'8 settembre del 1943, non esitò ad aderire al movimento della Resistenza. Non negò mai la sua adesione al fascismo durante gli anni della Marcia su Roma, eppure quest'esperienza gli servì per capire anche cosa fosse veramente questo movimento. Sabato Martelli fu forse uno dei primi - tra coloro che avevano appoggiato il fascismo - ad allontanarsi da esso, con la speranza di poter ricondurre il popolo italiano verso quella libertà che gli era stata sottratta. Con il senno di poi potremmo dire che il partigiano "Tevere" riuscì a portare a termine la sua missione, sebbene l'impresa gli costò la vita e un lungo silenzio.

${ }^{4}$ GRASSIA, Edoardo, Sabato Martelli Castaldi. Il generale partigiano, Milano, Mursia, 2016, pp. 12-13. 


\section{L'AUTORE}

Matteo TOMASONI ha conseguito il titolo di dottore di ricerca in Storia presso l'Universidad de Valladolid (Spagna, 2014), con una tesi sul fascismo spagnolo. Già dottore magistrale in Storia d'Europa presso l'Università di Bologna (2008), negli ultimi anni ha svolto attività di ricerca tra Spagna, Italia e Germania e collabora con vari gruppi fra cui il SIdIF (Seminario Interuniversitario de Investigadores del Fascismo), e la rivista «Zibaldone. Estudios italianos» di cui è membro della redazione. I suoi interessi sono rivolti allo studio dell'evoluzione storica del fascismo e dei movimenti politici del periodo tra le due guerre mondiali, oltre allo studio di alcuni aspetti della Prima Guerra Mondiale. Ha pubblicato El caudillo olvidado. Vida obra y pensamiento de Onésimo Redondo (1905-1936), Granada, Comares, 2017.

URL: < http://www.studistorici.com/progett/autori/\#Tomasoni > 